\title{
The Geometry of Power Systems Steady-State Equations- Part II: a Power Surface Study
}

\author{
B.I. Ayuev ${ }^{1}$, V.V Davydov ${ }^{2,3,}$, , P.M. Erokhin ${ }^{1,4}$, V.G. Neuymin ${ }^{5}$, A.V. Pazderin ${ }^{4}$
}

${ }^{1}$ JSC "System Operator of the Unified Power System," Moscow, Russia.

${ }^{2}$ Branch of JSC "System Operator of the Unified Power System" United dispatching department of the power system of Siberia, Kemerovo, Russia.

${ }^{3}$ East Siberia State University of Technology and Management, Ulan-Ude, Russia.

${ }^{4}$ Ural Federal University, Yekaterinburg, Russia.

${ }^{5}$ JSC "Scientific and Technical Center of Unified Power System", Saint Petersburg, Russia

\begin{abstract}
Steady-state equations play an essential part in the theory of power systems and the practice of computations. These equations are directly or mediately used almost in all areas of the theory of power system states, constituting its basis. This two-part study deals with a geometrical interpretation of steadystate solutions in a power space. Part I has proposed considering the power system's steady states in terms of power surface. Part II is devoted to an analytical study of the power surface through its normal vectors. An interrelationship between the entries of the normal vector is obtained through incremental transmission loss coefficients. Analysis of the normal vector has revealed that in marginal states, its entry of the slack bus active power equals zero, and the incremental transmission loss coefficient of the slack bus equals one. Therefore, any attempts of the slack bus to maintain the system power balance in the marginal state are fully compensated by associated losses. In real-world power systems, a change in the slack bus location in the marginal state makes this steady state non-marginal. Only in the lossless power systems, the marginal states do not depend on a slack bus location.
\end{abstract}

Index Terms: distributed slack bus, feasibility region, incremental transmission loss coefficient, Jacobian, marginal state, normal vector, power flow, power surface, power system, slack bus, steady state.

\footnotetext{
${ }^{*}$ Corresponding author.

E-mail: davv@bur.so-ups.ru
}

http://dx.doi.org/10.38028/esr.2020.03.0006

Received May 25, 2020. Revised June 19, 2020.

Accepted July 23, 2020. Available online December 27, 2020.

This is an open access article under a Creative Commons Attribution-NonCommercial 4.0 International License.

(C) 2020 ESI SB RAS and authors. All rights reserved.

\section{INTRODUCTION}

Steady-state models play a crucial part in the analysis, planning, and control of power systems. Therefore, an analytical investigation and understanding of steady-state equations are essential since they provide qualitative insights and facilitate the evolution of power system theories and elaboration of faster and more efficient algorithms. At present, most publications tend to use an algebraic approach. Mathematical expressions and equations are efficient tools for a numerical solution but are complicated for the qualitative analysis in the case of multidimensional systems. At the same time, a geometrical consideration allows taking advantage of powerful tools of modern geometry and obtaining noteworthy results. Part I [1] of the two-part study suggests the geometrical interpretation of a set of all the steady-state solutions in terms of surface in a power space. Consideration of the whole set of power system steady states using power surface allows taking advantage of differential geometry tools to qualitatively study steady-state models.

The objective of the present paper is to analytically investigate the power surface. This work improves upon the early study [2] considering the steady- states equations with distributed slack bus. The rest of the paper is organized as follows. In Section II, equations of a tangent plane and a normal vector to the power surface are obtained. Section III investigates the entries of the normal vector to the power surface and their specific features in marginal states of a power system. It also examines an interrelationship between entries of the normal vector to the power surface and entries of the normal vector to the surface of marginal states. Section IV considers the influence of a slack bus location on the marginal states through the entries of the normal vector to the power surface by the example of a 4-bus power system. Section $\mathrm{V}$ analyzes the adequacy of the parameters of marginal states obtained when using a distributed slack bus to assess an actual steady-state stability reserve of the 
power system in terms of power. Section VI presents the conclusions.

\section{TANGent Plane to Power Surface}

One of the differential geometry tools is tangent planes to the surface and their normal vectors. An equation of a tangent plane to the power surface is conveniently obtained by using nodal steady-state equations in polar coordinates:

$$
\begin{aligned}
& \Delta P(P, V, \delta)=0 ; \\
& \Delta P_{\mathrm{b}}\left(P_{b}, V, \delta\right)=0 ; \\
& \Delta Q(Q, V, \delta)=0 .
\end{aligned}
$$

where $P$ and $Q$ are an injection power vector consisting of the active power for buses other than the slack bus and the reactive power for $P Q$-specified buses, respectively; $\delta$ and $V$ are vectors defined by phase angles for buses other than the angle reference bus and voltage magnitude for $P Q$-specified buses, respectively; subscript $b$ refers to the slack bus.

Linearization of (1) gives:

$$
\left[\begin{array}{cc}
\frac{\partial \Delta P}{\partial \delta} & \frac{\partial \Delta P}{\partial V} \\
\frac{\partial \Delta P_{b}}{\partial \delta} & \frac{\partial \Delta P_{b}}{\partial V} \\
\frac{\partial \Delta Q}{\partial \delta} & \frac{\partial \Delta Q}{\partial V}
\end{array}\right]\left[\begin{array}{c}
\Delta \delta \\
\Delta V
\end{array}\right]+\left[\begin{array}{l}
d P \\
d P_{b} \\
d Q
\end{array}\right]=\left[\begin{array}{l}
0 \\
0 \\
0
\end{array}\right]
$$

Unlike standard linearized equations, which are used for steady-state calculations, system (2) explicitly includes the linearized equation of active power balance at slack bus $b$. Hence, the augmented Jacobian in (2) is rectangular; its number of rows is greater than the number of columns by one. According to the theory of linear algebra, rows of such matrix are linearly dependent [3]. Consequently, there exists a non-zero vector $\lambda=\left[\lambda^{P^{\mathrm{T}}}, \lambda_{b}^{P}, \lambda^{Q^{\mathrm{T}}}\right]$ satisfying the following equation:

$$
\left[\begin{array}{l}
\lambda^{P} \\
\lambda_{b}^{P} \\
\lambda^{Q}
\end{array}\right]^{\mathrm{T}}\left[\begin{array}{cc}
\frac{\partial \Delta P}{\partial \delta} & \frac{\partial \Delta P}{\partial V} \\
\frac{\partial \Delta P_{b}}{\partial \delta} & \frac{\partial \Delta P_{b}}{\partial V} \\
\frac{\partial \Delta Q}{\partial \delta} & \frac{\partial \Delta Q}{\partial V}
\end{array}\right]=\left[\begin{array}{l}
0 \\
0
\end{array}\right]
$$

Hence, multiplying the left-hand side of (2) by $\lambda^{\mathrm{T}}$, we obtain the following equation:

$$
\lambda^{P^{\mathrm{T}}} d P+\lambda_{b}^{P} d P_{b}+\lambda^{Q^{\mathrm{T}}} d Q=0 .
$$

Expression (4) is the equation of the tangent plane to the power surface in the space of the active power of the buses and the reactive power of $P Q$-specified buses. Therefore, (4) determines the vector $\lambda$ as a normal vector to the tangent plane, i.e., to the power surface. According to (3), the normal vector to the power surface can be obtained by specifying $\lambda_{b}^{P}$ and solving the set of linear equations:

$$
\left[\begin{array}{cc}
\frac{\partial \Delta P}{\partial \delta} & \frac{\partial \Delta P}{\partial V} \\
\frac{\partial \Delta Q}{\partial \delta} & \frac{\partial \Delta Q}{\partial V}
\end{array}\right]^{\mathrm{T}}\left[\begin{array}{l}
\lambda^{P} \\
\lambda^{Q}
\end{array}\right]=-\lambda_{b}^{P}\left[\begin{array}{l}
\frac{\partial \Delta P_{b}^{\mathrm{T}}}{\partial \delta} \\
\frac{\partial \Delta P_{b}^{\mathrm{T}}}{\partial V}
\end{array}\right]
$$

in which the coefficient matrix is the transposed power flow Jacobian.

\section{Study on the Normal Vector to Power Surface}

To determine an interrelationship between the entries of a normal vector to a power surface, it is convenient to use the system power balance equation

$$
P_{b}+\sum_{\forall k \neq b} P_{k}-\pi=0
$$

where $\pi$ is a system power loss.

The active power of the slack bus and power loss are dependent variables. Therefore, differentiation of (6) with respect to independent variables (power at specified buses) yields:

$$
\left[\frac{\partial P_{b}}{\partial P}\right]+e^{\mathrm{T}}-\left[\frac{\partial \pi}{\partial P}\right]=0^{\mathrm{T}} ; \quad\left[\frac{\partial P_{b}}{\partial Q}\right]-\left[\frac{\partial \pi}{\partial Q}\right]=0^{\mathrm{T}},
$$

where $e$ is the vector of all ones; $\left[\frac{\partial \pi}{\partial P}\right]$ and $\left[\frac{\partial \pi}{\partial Q}\right]$ are vectors of incremental transmission loss coefficients (ITLs). In turn, differentiation of (1) with respect to independent variables yields:

$$
\begin{aligned}
& \left|\begin{array}{ll}
\frac{\partial \Delta P}{\partial \delta} & \frac{\partial \Delta P}{\partial V} \\
\frac{\partial \Delta Q}{\partial \delta} & \frac{\partial \Delta Q}{\partial V}
\end{array}\right|\left[\begin{array}{ll}
\frac{\partial \delta}{\partial P} & \frac{\partial \delta}{\partial Q} \\
\frac{\partial V}{\partial P} & \frac{\partial V}{\partial Q}
\end{array}\right]+[\mathrm{E}]=[0] ; \\
& {\left[\frac{\partial \Delta P_{b}}{\partial P}\right]=\left[\frac{\partial P_{b}}{\partial P}\right]+\left[\frac{\partial \Delta P_{b}}{\partial \delta}\right]\left[\frac{\partial \delta}{\partial P}\right]+\left[\frac{\partial \Delta P_{b}}{\partial V}\right]\left[\frac{\partial V}{\partial P}\right]=0^{\mathrm{T}} ;} \\
& {\left[\frac{\partial \Delta P_{b}}{\partial Q}\right]=\left[\frac{\partial P_{b}}{\partial Q}\right]+\left[\frac{\partial \Delta P_{b}}{\partial \delta}\right]\left[\frac{\partial \delta}{\partial Q}\right]+\left[\frac{\partial \Delta P_{\mathrm{b}}}{\partial V}\right]\left[\frac{\partial V}{\partial Q}\right]=0^{\mathrm{T}},}
\end{aligned}
$$

where [E] and [0] are identity and zero matrices, respectively. In turn, the use of (8) in (9) yields:

$$
\left[\begin{array}{cc}
\frac{\partial \Delta P}{\partial \delta} & \frac{\partial \Delta P}{\partial V} \\
\frac{\partial \Delta Q}{\partial \delta} & \frac{\partial \Delta Q}{\partial V}
\end{array}\right]^{\mathrm{T}}\left[\begin{array}{l}
\frac{\partial P_{b}^{\mathrm{T}}}{\partial P} \\
\frac{\partial P_{b}^{\mathrm{T}}}{\partial Q}
\end{array}\right]=\left[\begin{array}{c}
\frac{\partial \Delta P_{b}^{\mathrm{T}}}{\partial \delta} \\
\frac{\partial \Delta P_{b}^{\mathrm{T}}}{\partial V}
\end{array}\right] .
$$

Comparison of (10) with (5) and use of (7) yield:

$$
\begin{gathered}
\lambda_{m}^{P}=\left(1-\frac{\partial \pi}{\partial P_{m}}\right) \lambda_{b}^{P} ; \\
\lambda_{m}^{Q}=-\frac{\partial \pi}{\partial Q_{m}} \lambda_{b}^{P} .
\end{gathered}
$$

The normal vector to the power surface does not depend on a selection of the slack bus because an alteration of the slack bus does not change solution to (5) if the entry of the normal vector of a new slack bus to set is equal to a value 
of this entry obtained by solving (5) using the old slack bus. Thus, (11)-(12) allow revealing the interrelationship between the ITLs obtained by using the new slack bus and the old one:

$$
\frac{\partial \pi_{k}}{\partial P_{m}}=\frac{\frac{\partial \pi_{b}}{\partial P_{m}}-\frac{\partial \pi_{b}}{\partial P_{k}}}{1-\frac{\partial \pi_{b}}{\partial P_{k}}} ; \quad \frac{\partial \pi_{k}}{\partial Q_{m}}=\frac{\frac{\partial \pi_{b}}{\partial Q_{m}}}{1-\frac{\partial \pi_{b}}{\partial P_{k}}},
$$

where $\frac{\partial \pi_{b}}{\partial P_{m}}$ and $\frac{\partial \pi_{k}}{\partial P_{m}}$ are ITLs obtained by using slack bus $b$ and new slack bus $k$, respectively.

Consider specific features of the normal vector to the power surface in a marginal state. According to (5) and (11)-(12), the entries of the normal vector can have different values. Assume that the normal vector entry $\lambda_{k}^{P}$ is zero at some point on the power surface. According to (11), this will only be when $1-\frac{\partial \pi}{\partial P_{k}}=0$, i.e.,

$$
\lambda_{k}^{P}=0 \Rightarrow \frac{\partial \pi}{\partial P_{k}}=1
$$

Assume that bus $k$ is assigned as a slack bus at this point. Then, the adjusted (5) can be represented as follows:

$$
\left[\begin{array}{cc}
\frac{\partial \Delta P}{\partial \delta} & \frac{\partial \Delta P}{\partial V} \\
\frac{\partial \Delta Q}{\partial \delta} & \frac{\partial \Delta Q}{\partial V}
\end{array}\right]^{\mathrm{T}}\left[\begin{array}{l}
\lambda^{P} \\
\lambda^{Q}
\end{array}\right]=-\lambda_{k}^{P}\left[\begin{array}{c}
\frac{\partial \Delta P_{k}^{\mathrm{T}}}{\partial \delta} \\
\frac{\partial \Delta P_{k}^{\mathrm{T}}}{\partial V}
\end{array}\right]=\left[\begin{array}{l}
0 \\
0
\end{array}\right] .
$$

The matrix in (15) is the transposed power flow Jacobian by using slack bus $k$, and condition (15) determines its singularity, i.e., a marginal state. According to (14), in the marginal state, the incremental transmission loss coefficient of the slack bus is equal to one, which is why any attempts of the slack bus to maintain the power system steady state are compensated by associated power loss. The inability of the slack bus to maintain the steady state even at some buses determines the power system marginal state. In the marginal state, the slack bus as if loses connection to a part or the whole of the power system; it corresponds to the absence of slack bus in the computational model of steady states with all consequences.

The interpretation of the marginal state surface in the subspace of power at specified buses as the boundary of the power surface projection along the axis of slack bus

Table 1. A 4-bus power system.

\begin{tabular}{ccccc}
\hline \hline Bus & 1 & 2 & 3 & 4 \\
\hline \multicolumn{5}{c}{ Base Case } \\
\hline$P$ & 20 & -50 & 50 & -22.1951 \\
\hline$\lambda^{P}$ & 0.9259 & 0.9069 & 1.0231 & 1 \\
\hline \multicolumn{5}{c}{ Marginal states obtained by using slack bus 4 } \\
\hline$P$ & 20 & -411.77 & 411.77 & -465.27 \\
\hline$\lambda^{P}$ & -1 & -0.9794 & 0 & 0 \\
\hline \multicolumn{5}{c}{ Marginal states obtained by using slack bus 1 } \\
\hline$P$ & -118.72 & -171.51 & 171.51 & -22.1951 \\
\hline$\lambda^{P}$ & 0 & 0 & 1 & 0.9775 \\
\hline \hline
\end{tabular}

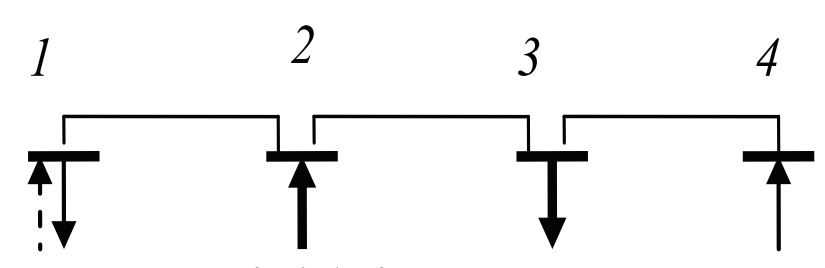

Fig. 1. A 4-bus power system.

active power onto the subspace of power of all other buses allows obtaining an interesting interrelationship between the normal vector to the power surface in marginal state and the normal vector to the marginal state surface. According to (14), the slack bus entry of the normal vector to the power surface is equal to zero in the marginal state. Therefore, in the marginal state, the normal vector to the power surface is orthogonal to the axis of slack bus active power and will be projected onto the subspace of power at specified buses in full size. Consequently, the entries of the normal vector to the marginal state surface will be equal to the corresponding entries of the normal vector to the power surface.

\section{INFLUENCE OF SLACK BUS LOCATION ON MARGINAL STATE}

In the marginal state, in the case of a changing slack bus, generally, condition (15) will not be satisfied, unless a new slack bus has zero entry of the normal vector to the power surface in (5). A simple system with $4 P V$ buses in Fig. 1 allows showing this.

Table 1 indicates the power at buses in MW (the minus sign corresponds to generation) and entries of the normal vector to the power surface for the base case and also for the marginal states obtained by using slack bus 4 , and slack bus 1 . These marginal states are the results of a generation increase at bus 2 and a load increase at bus 3 . The line parameters are $Z_{12}=Z_{34}=5+j 10 \Omega, Z_{23}=20+j 40 \Omega$, and $V_{1}=V_{2}=V_{3}=V_{4}=110 \mathrm{kV}$.

Consider the parameters of the marginal state obtained by using slack bus 4 . Since the power transfer through line 2-3 is associated with loss, slack bus 4 supports the power transfer through line 3-4. The marginal state occurs when line 2-3 is overloaded. The steady state remains marginal if bus 3 is assigned as slack bus since its entry of the normal vector to the power surface is also equal to zero in this case. However, it will not remain marginal, if the slack bus is moved to bus 1 as its entry of the normal vector is not equal to zero and there is a possibility of continuing generation increase at bus 2 and load increase at bus 3 . In the marginal state, the ITLs for bus 4, obtained by using slack bus 1 or 2 , are equal to one.

The marginal state will be different if slack bus 1 is used because it maintains the power system steady state through line 1-2 in this case. The ITL for the slack bus increases with growth in the power transfer. As soon as it reaches one, the load increase at bus 3 becomes impossible. Slack bus 1 is unable to maintain the steady state of bus 3 and 4 


\begin{tabular}{ccccc}
\multicolumn{5}{c}{ TABLE 2. 4-Bus LOSSLESS POWER SYSTEM. } \\
\hline \hline Bus & 1 & 2 & 3 & 4 \\
\hline \multicolumn{5}{c}{ Base Case } \\
\hline$P$ & 20 & -50 & 50 & -20 \\
\hline$\lambda^{P}$ & 1 & 1 & 1 & 1 \\
\hline \multicolumn{5}{c}{ Marginal states obtained by using slack bus 4} \\
\hline$P$ & 20 & -322.5 & 322.5 & -20 \\
\hline$\lambda^{P}$ & 1 & 1 & 0 & 0 \\
\hline \multicolumn{7}{c}{ Marginal states obtained by using slack bus 1 } \\
\hline$P$ & 20 & -322.5 & 322.5 & -20 \\
\hline$\lambda^{P}$ & 0 & 0 & 1 & 1 \\
\hline \hline
\end{tabular}

in this marginal state. The marginal state remains marginal if bus 2 becomes slack bus, as its entry of the normal vector is equal to zero. This steady state will not be marginal if the slack bus is bus 4 or 3 since these buses have non-zero entries of the normal vector. The marginal values of power at buses and system loss in the marginal state obtained by using slack bus 1 are smaller than those obtained by using slack bus 4 . At the same time, the marginal state obtained by using slack bus 4 and the steady states in its vicinity will be unstable if the dynamic Jacobian is used for the steadystate stability assessment [4].

The influence of the slack bus location on the marginal state can be explained by considering the power surface. Each power system steady state corresponds to a certain point on the power surface and vice versa. The projection of the power surface along the axis direction of the slack bus active power onto the subspace of power of all specified buses is the power flow feasibility region [1]. The boundary of this projection corresponds to a set of marginal states. Since the power surface is usually not plane, the points on the power surface, which correspond to the boundary of such a projection, i.e., marginal state, will be different for another slack bus. Therefore, the slack bus change in the marginal state makes the steady state non-marginal.

In the case of no resistances in the network in Fig. 1, the power system marginal states obtained by using slack bus 4 and slack bus 1 will be the same. The base case and marginal state parameters for the 4-bus lossless system with $Z_{12}=Z_{34}=j 10 \Omega, Z_{23}=j 40 \Omega$ are shown in Table II.

In any lossless power system, marginal states remain marginal regardless of the slack bus location. It can be shown if to consider the following. Firstly, if all the resistances are zero, the vector $\lambda=\left[e^{\mathrm{T}}, 1,0^{\mathrm{T}}\right]$ will always correspond to (3), i.e., it will always be the normal vector to the power surface. Secondly, in the marginal state, the dimension of a null space of the transposed augmented Jacobian (3) is equal to two [5]. Therefore, in the marginal state, another normal vector $\left[\lambda^{P^{T}}, 0, \lambda^{Q^{\mathrm{T}}}\right]$ to the power surface of the null space of transposed augmented Jacobian (3) will correspond to (14)-(15). Hence, in the marginal state, vector $\lambda$ of a linear combination of vectors

$$
\lambda=\beta\left[e^{\mathrm{T}}, 1,0^{\mathrm{T}}\right]^{\mathrm{T}}+\left[\lambda^{P^{\mathrm{T}}}, 0, \lambda^{Q^{\mathrm{T}}}\right]^{\mathrm{T}}
$$

will also be a normal vector to the power surface. Two cases are possible when new slack bus $k$ is used. If $\lambda_{k}^{P}=0$, then the entries of the normal vector remain unchanged in (14)-(15). Otherwise, the normal vector $\lambda$ (16) with $\beta=-\lambda_{k}^{P}$ will also correspond to the power flow Jacobian (15) singularity.

The fact that the marginal state does not depend on the slack bus location can also be explained by considering the power surface of a lossless power system. This power surface is plane [1] since it also satisfies the equation $\sum_{\forall k} P_{k}=0$. The points on the plane power surface that correspond to the boundaries of its projections along the active power axis direction of any slack bus onto the subspace of power of specified buses, i.e., the marginal states, are the same. Therefore, marginal states do not depend on the slack bus location. It is also noteworthy, that one side of this plane power surface corresponds to aperiodic stable steady states; while the other side is unstable ones, i.e., the same point on the power surface of the lossless power system corresponds to stable and unstable power system steady states [1].

\section{Distributed Slack Bus}

Several power flow programs use a so-called distributed slack bus, i.e., active power is balanced by several generators with specified participation factors [6]. Participation factors $\vartheta_{k}$ may be specified according to economic reasons, or based on requirements of the primary or secondary frequency control, and so on. In this case, a dependent variable $P^{S}$ is used for the distributed slack bus, which is taken into consideration in steady-state equations as $\vartheta_{k} P^{S}$ with $\sum_{k} \vartheta_{k}=1$, and the power flow Jacobian is represented as follows:

$$
\left[\begin{array}{ccc}
\frac{\partial \Delta P}{\partial \delta} & \frac{\partial \Delta P}{\partial V} & \vartheta \\
\frac{\partial \Delta P_{b}}{\partial \delta} & \frac{\partial \Delta P_{b}}{\partial V} & \vartheta_{b} \\
\frac{\partial \Delta Q}{\partial \delta} & \frac{\partial \Delta Q}{\partial V} & 0
\end{array}\right]
$$

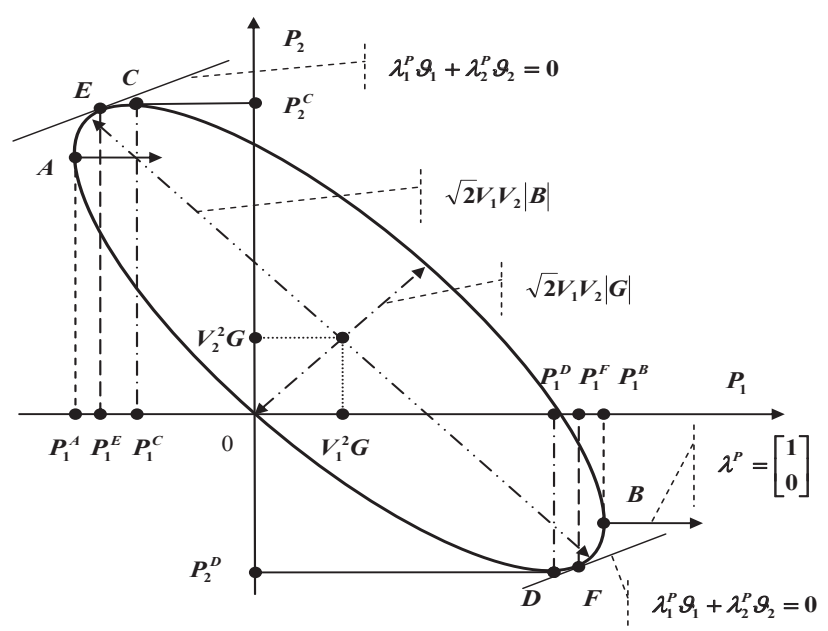

Fig. 2. Power ellipse of a 2-bus power system and marginal states 
Matrix (17) includes partial derivatives of active power balance equations for all buses, and subscript $b$ refers to a reference bus. In the marginal state, this matrix is singular. With the left-hand side eigenvector (corresponding to its zero eigenvalue) denoted by $\gamma$, singularity conditions of (17) can be represented as follows:

$$
\begin{gathered}
\vartheta^{\mathrm{T}} \gamma=\sum_{k \in S} \vartheta_{\mathrm{k}} \gamma_{k}^{P}=0 ; \\
{\left[\begin{array}{c}
\gamma^{P} \\
\gamma_{b}^{P} \\
\gamma^{Q}
\end{array}\right]^{\mathrm{T}}\left[\begin{array}{cc}
\frac{\partial \Delta P}{\partial \delta} & \frac{\partial \Delta P}{\partial V} \\
\frac{\partial \Delta P_{b}}{\partial \delta} & \frac{\partial \Delta P_{b}}{\partial V} \\
\frac{\partial \Delta Q}{\partial \delta} & \frac{\partial \Delta Q}{\partial V}
\end{array}\right]=\left[\begin{array}{l}
0 \\
0
\end{array}\right]^{\mathrm{T}} .}
\end{gathered}
$$

In turn, (19) can be rewritten as:

$$
\left[\begin{array}{cc}
\frac{\partial \Delta P}{\partial \delta} & \frac{\partial \Delta P}{\partial V} \\
\frac{\partial \Delta Q}{\partial \delta} & \frac{\partial \Delta Q}{\partial V}
\end{array}\right]^{\mathrm{T}}\left[\begin{array}{c}
\gamma^{P} \\
\gamma^{Q}
\end{array}\right]=-\gamma_{b}^{P}\left[\begin{array}{l}
\frac{\partial \Delta P_{b}^{\mathrm{T}}}{\partial \delta} \\
\frac{\partial \Delta P_{b}^{\mathrm{T}}}{\partial V}
\end{array}\right] .
$$

Comparison of (20) with (5) shows that vector $\gamma$ is the normal vector $\lambda$ to the power surface. Therefore, condition (18) can be represented as follows:

$$
\vartheta^{\mathrm{T}} \lambda=\sum_{k \subset S} \vartheta_{k} \lambda_{k}^{P}=0 .
$$

Thus, the marginal state determined by using the distributed slack bus corresponds to such points on the power surface at which their normal vectors to the power surface $\lambda$ are orthogonal to the vector of bus participation factors for balancing active power $\vartheta$. In the case of a 2-bus power system with $P V$-buses, two points $E$ and $F$ in Fig. 2 satisfy this condition.

Fig. 2 also shows the marginal states and power flow feasibility regions when the slack bus is bus 2 (the points $A, B$ and the straight line $\left[P_{1}^{A}, P_{1}^{B}\right]$ ) or bus 1 (the points $C$,

$D$ and the straight line $\left.\left[P_{2}^{C}, P_{2}^{D}\right]\right)$. This means that when the distributed slack bus is used, the obtained marginal states are not on the boundary of the power flow feasibility region but inside the region. Therefore, the steady-state stability reserve in terms of power, obtained by using the distributed slack bus, can be less than the reserve obtained by using the single slack bus. Consider, for example, the marginal state at point $E$. Indeed, when the marginal state is determined by changing load at bus 1 and using the distributed slack bus then, according to the arc $\mathrm{O} A E$ of the ellipse, at the beginning of the process, the power of bus 1 will gradually increase to $P_{1}^{A}$ and then decrease to $P_{1}^{E}$ . This means that the marginal state $E$ obtained by using the distributed slack bus will be farther in an angle space than the marginal state $A$ obtained by using single slack bus 2 but nearer in the power space. Usually, the steadystate stability reserve of a power system is determined in terms of power. Therefore, the steady-state stability reserve will be assessed more correctly if we use parameters of the steady state corresponding to point $A$, i.e., the parameters of the marginal state obtained by using single slack bus 2 .

Consideration of the system power balance equation

$$
P^{S}+\sum_{\forall k} P_{k}-\pi=0
$$

and use of an approach similar to (7)-(12) allow obtaining the following relationship between the entries of the normal vector to the power surface determined by using the distributed slack bus [5]:

$$
\begin{gathered}
\lambda_{m}^{P}=\left(1-\frac{\partial \pi^{S}}{\partial P_{m}}\right) \lambda^{S} ; \\
\lambda_{m}^{Q}=-\frac{\partial \pi^{S}}{\partial Q_{m}} \lambda^{S},
\end{gathered}
$$

where $\frac{\partial \pi^{S}}{\partial P_{m}}$ is ITL for bus $m$ determined by using the distributed slack bus $s$, and

$$
\lambda^{S}=\vartheta^{\mathrm{T}} \lambda=\sum_{k \in S} \vartheta_{k} \lambda_{k}^{P}
$$

Any changes in the distributed slack bus, even in marginal state when

$$
\lambda^{S}=0
$$

do not change the entries of the normal vector to the power surface as they correspond to (3). Therefore, if in the marginal state, the use of (25) gives $\lambda^{\text {Snew }} \neq 0$ for new distributed slack bus $s_{\text {new }}$, then according to (23)

$$
\lambda_{m}^{P}=\left(1-\frac{\partial \pi^{\text {Snew }}}{\partial P_{m}}\right) \lambda^{\text {Snew }},
$$

substitution of which into (25) and considering (26) yield:

$$
\frac{\partial \pi^{\text {Snew }}}{\partial P^{S}}=\sum_{m} \frac{\partial \pi^{\text {Snew }}}{\partial P_{m}} \vartheta_{m}=1 .
$$

Thus, in the marginal state, the incremental transmission loss coefficient of the distributed slack bus is equal to unity, which is why any manipulations of the distributed slack bus to balance active power are compensated by power loss caused by such manipulations. It means that the distributed slack bus is unable to maintain such a steady state. Any random insignificant power changes at buses in an unfavorable direction cannot be balanced by the distributed slack bus.

As in the case of the single slack bus, a change in the distributed slack bus (for example, an alteration in participation factors) in the marginal state makes this steady state non-marginal. Exceptions are the cases either where condition (21) is also satisfied in this steady state for a new structure of the distributed slack bus or where the lossless power system model is used. In the latter case, the normal vector (16) with $\beta=-\sum \vartheta_{k} \lambda_{k}^{P}$ corresponds to (18)-(21), i.e., the power flow Jacobian remains singular.

\section{CONCLUSION}

Part II of the two-part study investigates the power surface proposed in Part I. The findings have revealed that the entries of the normal vector to the power surface 
are interrelated through incremental transmission loss coefficients. Analysis of the normal vector to the power surface has shown that in marginal states of the power system the entry of the normal vector corresponding to the slack bus active power equals zero, and the incremental transmission loss coefficient of the slack bus is equal to one. Therefore, in marginal states, any attempts of the slack bus to balance active power in the power system are completely compensated by active power loss caused by such manipulations. The inability of the slack bus to maintain the steady state even of some buses determines the power system marginal state. In marginal states, the slack bus as if loses connection to a part or the whole of the power system; it corresponds to the absence of the slack bus in the computational steady-state model with all consequences. In real-world power systems, a change in the slack bus location in a marginal state makes this steady state non-marginal. Only in the lossless power systems, marginal states do not depend on the slack bus location. The study has also revealed that parameters of marginal states obtained by using the distributed slack bus may give an underestimation of the real steady-state stability reserve of the power system in terms of power.

\section{REFERENCES}

[1] B.I. Ayuev, V.V. Davydov, P.M. Erokhin, V.G. Neuymin, A.V. Pazderin, "Geometry of power system steady states equations - Part I: Power surface," Energy System Research., Submitted for publication.

[2] V.V. Davydov, P.M. Erokhin, "Studying power hypersurface of power system steady states," (in Russian) Proceedings of Irkutsk State Technical University, Irkutsk, Russia, no. 1(23), pp. 101-106, 2019.

[3] R.A. Horn, C.R. Johnson, Matrix Analysis. Cambridge, MA, USA: Cambridge Univ. Press, 1986.

[4] V.V. Davydov, P.M. Erokhin, M.A. Prudov, "Analysis of power system models for steady-state stability reserve assessment," Proc. $5^{\text {th }}$ Int. Sci.\&Tech. Conf. Power Industry by Youth Eyes (in Russian), Tomsk, Russia, 2014, vol. 1, pp. 67-70.

[5] V.V. Davydov, Research and Development of Calculation Models of Marginal States of Power Systems. A thesis presented to the Novosibirsk State Technical University in fulfillment of the thesis requirement for the degree of Doctor of Technical Sciences in Electrical Engineering (in Russian). Ulan-Ude, Russia: 2019, p. 464. [Online]. Available at: https://www.nstu.ru/science/dissertation_sov/ dissertations/view?id=17561 (accessed....).

[6] A.G. Exposito, L.M. Ramos, J.R. Santos, "Slack bus selection to minimize the system power imbalance in load-flow studies," IEEE Trans. Power Syst., vol. 19, no. 2, pp. 987-995, May 2004.

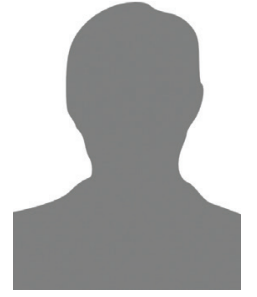

Boris I. Ayuev received the Dipl. Eng. and the Ph.D. degree in electrical engineering from Ural Federal University, Yekaterinburg, Russia, in 1979 and 1999 respectively, and Dr.Sc. degree in electrical engineering from Novosibirsk State Technical University, Novosibirsk, Russia in 2008.

$\mathrm{He}$ is now the Chairman of the JSC System Operator of the United Power System, Moscow, Russia. His research interests include control, operations, planning, and economics of power systems.

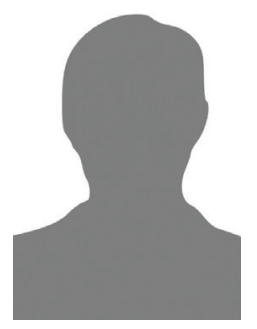

Viktor V. Davydov received the Dipl. Eng. in electrical engineering from East Siberian Technological Institute, Ulan-Ude, Russia, in 1979, the Ph.D. degree in electrical engineering from Ural Federal University, Yekaterinburg, Russia, in 1987, and the Dr.Sc. degree in electrical engineering from Novosibirsk State Technical University, Novosibirsk, Russia in 2019.

He is now a leading specialist of the JSC System Operator of the United Power System branch - ODU of Siberia, UlanUde, Russia. His research interests are steady state computing models, power system dynamics, control and stability, marginal states, and emergency control.

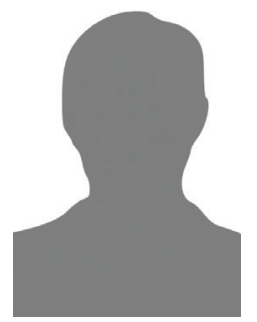

Petr M. Erokhin received the Dipl. Eng., the Ph.D. and Dr.Sc. degrees in electrical engineering from Ural Federal University, Yekaterinburg, Russia in 1969, 1975, and 2005 respectively.

$\mathrm{He}$ is now a Professor of Electrical Engineering at Ural Federal University, Yekaterinburg, Russia. His research interests include control, operations, planning, and economics of power systems. 


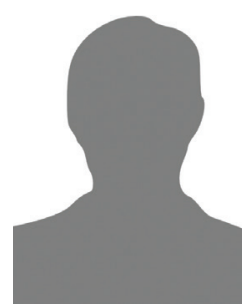

Vladimir G. Neuymin received the Dipl.Eng. and the Ph.D. degree in electrical engineering from Ural Federal University, Yekaterinburg, Russia in 1982, 1987, respectively.

He is now a Head of Department of JSC "Scientific and Technical Center of Unified Power System," Yekaterinburg, Russia. His research interests include control and stability, steady states computing models, power system dynamics, state estimation, planning, and economics of power systems.

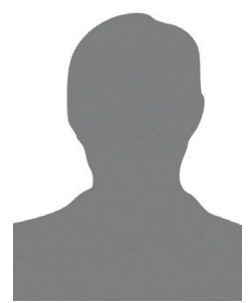

Andrey V. Pazderin received the Dipl. Eng., the Ph.D. and Dr.Sc. degrees in electrical engineering from Ural Federal University, Yekaterinburg, Russia in 1982, 1987, and 2005 respectively.

$\mathrm{He}$ is now a Professor of Electrical Engineering at Ural Federal University, Yekaterinburg, Russia. His research interests include control and stability, operations, state estimation, planning, and economics of power systems. 